\title{
On the Optimization of Multi-Cell SLIPT Systems
}

\author{
Amr M. Abdelhady, Osama Amin, Basem Shihada, and Mohamed-Slim Alouini \\ Computer Electrical, and Mathematical Science and Engineering (CEMSE) Division, \\ King Abdullah University of Science and Technology (KAUST), Thuwal, Makkah Province, Kingdom of Saudi Arabia, \\ E-mail: $\{$ amr.abdelhady, osama.amin, basem.shihada, slim.alouini $\} @$ kaust.edu.sa.
}

\begin{abstract}
In this paper, we study the performance of simultaneous lightwave information and power transfer (SLIPT) systems of multi-cell indoor scenario. We aim to investigate the energy harvesting and data rate performance of multiple users while meeting the lightning constraints. To this end, we develop optimization frameworks and tune the light emitting diodes average currents to improve the performance of the SLIPT system. Firstly, we propose an algorithm to maximize the spectral efficiency (SE) subject to lighting and minimum harvested energy per user requirements. The proposed algorithm can be implemented in a distributed fashion with a reduced computational burden at each node. Then, we consider the energy harvesting maximization problem to investigate the maximum possible energy gain and its corresponding SE performance. Finally, we present some extensive simulations to explore the benefit of the optimization frameworks with respect to standard equal allocation setting. In addition, we monitor the effect of changing several system parameters on the two objectives and highlight the underlying trade-off between them.
\end{abstract}

\section{INTRODUCTION}

In recent decades, the over congestion of the radio frequency (RF) spectrum, and the ever-growing users' wireless data traffic, have thrust research efforts to exploit higher frequency bands in the electromagnetic spectrum [1]. Visible light communication (VLC) has received lots of attention in both academia and industry as it possesses many promising features. VLC systems offer large bandwidth, physical security, great potential in using existing lighting infrastructure, and low cost solutions. These features have nominated VLC to be one of the candidates for $5 \mathrm{G}$ access technologies [2].

VLC systems can offer many services besides illumination and communications. For example, energy can be harvested from light and used to support devices operating with limited energy sources. Energy consumption at the user equipment has become a very important aspect in new wireless communications networks especially with the evolution of Internet of things. Thus, it is imperative to analyze and optimize the performance of different services.

Light energy harvesting has been recently investigated for VLC systems considering different system configurations [3][9]. In [4], Rakia et al. considered a hybrid VLC/RF energy harvesting enabled relaying system and optimized the VLC transmitter DC bias to maximize the average system data rate. In the context of multiple users, Zenaidi et al. studied the achievable rate region of a hybrid VLC/RF system where two transmitters are communicating with two designated receivers through a relay [3]. In [5], [6], the authors investigated the resource allocation problem to maximize the spectral efficiency
(SE) of a single cell VLC downlink system with energy harvesting capabilities. Motivated by the aforementioned results, the authors in [7] studied the trade-off between illumination service and communications service via multi-objective optimization while imposing a per-user energy harvesting constraint. Moreover, Liu at al. demonstrated experimentally a VLC receiver using solar cell, which is used in both energy harvesting and data detection [9]. The performance is measured practically in terms of the error probability and generated voltage versus illuminance. With the increased research interest in VLC systems with energy harvesting capabilities, Diamantoulakis et al. introduced the "SLIPT" term and proposed different operation policies to balance the trade-off between the amount of harvested energy and communications quality of service for a single user single cell scenario [8].

In this paper, we study the performance optimization of multi-cell SLIPT system that is used to serve multiple users. The multi-cell scenario introduces interference on the served users, which adds a challenge on the SLIPT performance optimization. As such, we first optimize the LEDs average current to maximize the SE subject to both illumination and energy harvesting constraints. In this context, we propose a lowcomplexity algorithm that can be implemented in a distributed way to reduce the computational burden on different LED nodes. Then, we consider the energy harvesting maximization problem and propose a concave lower bound for the objective function and solve it. Finally, we show the gains achieved by optimization over simple equal allocation strategies on both objectives and compare the losses in SE/energy harvesting performance when energy harvesting/SE is optimized.

\section{System MODEL}

Consider a SLIPT system with $K$ transmitters that communicate with $K$ receivers simultaneously using the same frequency band $^{1}$. Thus, each user is exposed to interference from at most $K-1$ undesired transmitted streams. We assume perfect knowledge of channel state information at the transmitter LEDs. The received signal at the $i$-th user can be expressed as

$$
y_{i}=\sum_{j=1}^{K} h_{i, j} s_{j}+n_{i},
$$

\footnotetext{
${ }^{1}$ The solutions presented in this work are general enough to handle systems having $N \leq K$ receivers, with one-to-one mapping where the surplus users' links in our model can be assigned zero-channel gains.
} 


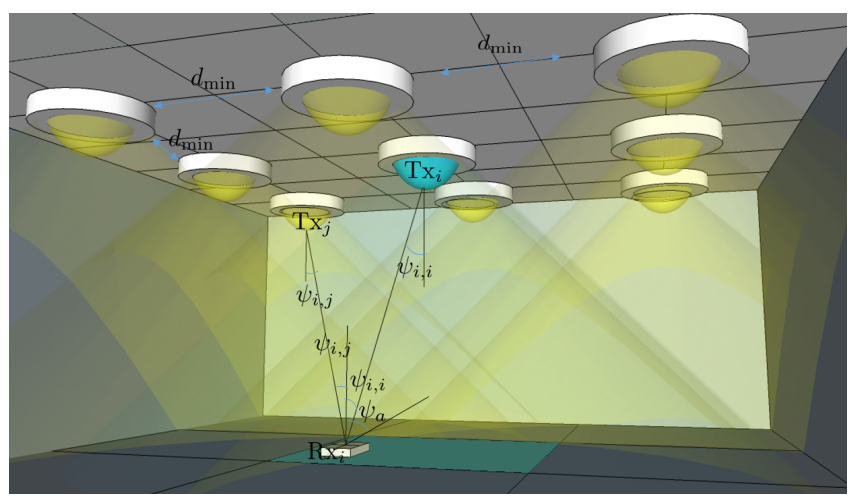

Fig. 1: System Model

where $s_{i}$ is the current intensity used to transmit a symbol to the $i$-th user by the $i$-th transmitter, $h_{i, j}$ is the VLC channel gain coefficient between the $j$-th transmitter and the $i$-th receiver, and $n_{i}$ represents the $i$-th receiver noise which is modeled as zero mean additive white Gaussian noise with variance $\sigma_{n}^{2}$. The direct link that represents the intended transmission established between the transmitter and its corresponding user has the same indices, i.e., $h_{i, i}$.

Throughout this work, we consider upper and lower bounds on illuminance at the receivers' plane as imposed by the lighting constraints. We define the illuminance lower bound to be the least possible illuminance within a cell coverage when all other sources are turned off. As for the upper bound, we define it as the sum of the maximum illuminances of a cluster of neighboring transmitters within their cell coverages. A cluster is defined to be a set of transmitters that are mutually separated by less than $2 d_{\min }$ inter-distance, where $d_{\min }$ is the minimum distance between any two transmitters in the setup. We denote the total number of transmitter clusters by $|\Phi|$ in the system, with $\Phi$ being the set of all possible clusters in the system. It can be noticed that $\Phi$ depends mainly on the cells deployment layout. We assume that transmitters are placed on the ceiling in a uniform pattern, as shown in Fig. 1. Furthermore, all users are assumed to be able to harvest energy during the whole transmission frame while decoding operations are done in parallel [10]. We impose a constraint on the system to achieve a minimum amount of harvested energy for each user. Finally, the total transmitted power by all cells is constrained to be less than the available power budget $P_{\mathrm{M}}$.

We assume line-of-sight existence for all links and neglect any reflected components, we employ the channel model in [11] where the channel gain $h_{i, j}$ between transmitter $j$ and receiver $i$ depends on the position of the receiver with respect to the transmitter, and is given by:

$$
h_{i, j}=\frac{(m+1) R_{\mathrm{PD}} A_{\mathrm{PD}}}{2 \pi d_{i, j}^{2}} \cos ^{m+1}\left(\psi_{i, j}\right) \operatorname{rect}\left(\frac{\psi_{i, j}}{\psi_{\mathrm{a}}}\right),
$$

where $m=-\ln 2 / \ln \left(\cos \left(\phi_{\mathrm{a}}\right)\right)$ is the Lambertian order, $\phi_{\mathrm{a}}$ is the semi-angle at half-power of the light source emission pattern, $A_{\mathrm{PD}}$ is the effective photo-detector area, $d_{i, j}$ is the distance between the $j$-th transmitter and user $i, \psi_{i, j}$ is the angle between the incident light ray from transmitter $j$ and the normal to the $i$-th photo-detector plane, $\psi_{\mathrm{a}}$ is the field of view of the user's receiver (assumed to be constant for all used receivers), and $\operatorname{rect}(x)$ is the rectangular function defined as $\operatorname{rect}(x)=1$ if $|x| \leq 1$, and 0 otherwise.

\section{SE VS ENERGY HARVESTING MAXIMIZATION}

In this section, we study the resource allocation problems of a multiple user SLIPT system in an interference-based scenario where the SE and energy harvesting maximization are of particular interest. The receivers are assumed to treat interference as noise at detection. The exact expression for VLC channel capacity is not yet available, consequently, we express the system overall SE based on VLC channels capacity lower bound [12] with $s_{i} \sim \operatorname{Exp}\left(1 / x_{i}\right)$ as

$$
\eta_{\mathrm{SE}}=\frac{1}{2} \sum_{i=1}^{K} \log _{2}\left(1+\frac{e}{2 \pi} \frac{\gamma_{i, i} x_{i}^{2}}{1+\sum_{j=1, j \neq i}^{K} \gamma_{i, j} x_{j}^{2}}\right),
$$

where $\gamma_{i, j}$ is the VLC channel-to-noise ratio of the link between the $j$-th transmitter and the $i$-th receiver defined as $\gamma_{i, j}=h_{i, j}^{2} / \sigma_{n}^{2} \forall i \neq j, \gamma_{i, i}=h_{i, i}^{2} / \sigma_{n}^{2}, x_{i}=\mathbb{E}\left\{s_{i}\right\}$.

On the other hand, we evaluate the energy harvesting performance through the total harvested power by all receivers which can be expressed as [4]

$$
P_{\mathrm{H}, \text { tot }}=\sum_{i=1}^{K} P_{\mathrm{H}, i},
$$

where, $P_{\mathrm{H}, i}$ is the total harvested power by the $i$-th receiver calculated as [4, Eq. 4]

$P_{\mathrm{H}, i}=0.75 V_{\mathrm{t}} \sum_{j=1}^{K} h_{i, j} x_{j} \ln \left(1+\sum_{j=1}^{K} h_{i, j} x_{j} / I_{\mathrm{o}}\right)$,

where $R_{\mathrm{PD}}$ is the photodetector responsivity, $V_{\mathrm{t}}$ is the thermal voltage and $I_{\mathrm{O}}$ is the dark saturation current of the photodetector.

\section{A. SE Maximization}

The considered resource allocation problem tunes the average current intensity vector $\boldsymbol{x}=\left\{x_{i}\right\}_{i=1}^{K}$ to maximize the total downlink SE. The design should maintain the required illumination level within range, satisfy the transmitted power budget and maintain minimum harvested energy per user. Accordingly, we formulate the following optimization problem:

(P1)

$$
\begin{array}{ll}
\max _{\boldsymbol{x}} & \eta_{\mathrm{SE}} \\
\text { subject to } & \mathrm{C} 1: \sum_{i=1}^{K} \kappa x_{i}^{2} \leq P_{\mathrm{M}} \\
& \mathrm{C} 2: x_{i} \eta_{\phi} h_{\min } / R_{\mathrm{PD}} \geq E_{\min } \quad \forall i \\
& \mathrm{C} 3: \sum_{i \in \mathcal{N}_{j}} x_{i} \eta_{\phi} h_{\max } / R_{\mathrm{PD}} \leq E_{\max } \quad \forall j, \\
& \mathrm{C} 4: P_{\mathrm{H}, i} \geq P_{\mathrm{H}, \mathrm{th}} \quad \forall i,
\end{array}
$$


where $\mathrm{C} 1$ satisfy the transmitter total radiated power budget with $\kappa$ denoting the ratio between the LED electrical power usage and the square of its driving electric current.

The constraints $\mathrm{C} 2$ and $\mathrm{C} 3$ ensure the minimum and maximum allowable illumination levels $E_{\min }$ and $E_{\max }$, respectively, with $h_{\min }$ and $h_{\max }$ being the channel gain at the furthest/nearest point from the transmitter within cell coverage, $\eta_{\phi}$ representing the ratio between the luminous flux emitted by the LED and its driving current and $\mathcal{N}_{l}$ representing the $l$-th transmitters cluster in the constellation. $\mathrm{C} 4$ is used to guarantee minimum harvested energy by each user. Interestingly, $P_{\mathrm{H}, i}$ is positive and monotonically increasing with respect to $\sum_{j=1}^{K} h_{i, j} x_{j}$, thus we can rewrite $\mathrm{C} 4$ equivalently as

$$
\sum_{j=1}^{K} h_{i, j} x_{j} \geq g^{-1}\left(P_{\mathrm{H}, \mathrm{th}}\right),
$$

where $g^{-1}(x)$ is the inverse function of $g(x)=$ $0.75 V_{\mathrm{t}} x \ln \left(1+x / I_{\mathrm{o}}\right)$.

To solve this problem, we employ alternate optimization where one of the variables is tuned while the rest are kept fixed, such that the updated solution maintains the feasibility conditions and the overall objective function is always improved. Then, roles are changed in a cyclic order between the variable being optimized and the others till convergence is reached. Thus, in each iteration of the alternate optimization procedure we need to solve a single variable optimization problem of the following form:

$$
\begin{array}{ll}
\max _{x_{a}} & \eta_{\mathrm{SE}}\left(x_{a}\right) \\
\text { subject to } & x_{\min , a} \leq x_{a} \leq x_{\max , a},
\end{array}
$$

where $x_{\min , a}$ is defined as

$x_{\min , a}=\max \left(\frac{E_{\min } R_{\mathrm{PD}}}{\eta_{\phi} h_{\min }}, \frac{g^{-1}\left(P_{\mathrm{H}, \mathrm{th}}\right)-\sum_{j \neq a} x_{j} h_{a, j}}{h_{a, a}}\right)$

while $x_{\max , a}$ is defined as

$x_{\max , a}=\min \left(\sqrt{P_{\mathrm{M}} / \kappa-\sum_{i \neq a} x_{i}^{2}}, \frac{R_{\mathrm{PD}} E_{\max }}{\eta_{\phi} h_{\max }}-\sum_{\substack{i \in \mathcal{N}_{a} \\ i \neq a}} x_{i}\right)$.

The lower limit of (P2) constraint is imposed by the virtue of $\mathrm{C} 2$ and $\mathrm{C} 4$, while the upper constraint limit is imposed by $\mathrm{C} 1$ and $\mathrm{C} 3$. As for $\eta_{\mathrm{SE}}\left(x_{a}\right)$, it is defined as

$$
\begin{aligned}
& \eta_{\mathrm{SE}}\left(x_{a}\right)=\frac{1}{2 \ln (2)}\left(\sum_{i=1}^{K} \ln \left(1+\sum_{j \neq a} \tilde{\gamma}_{i, j} x_{j}^{2}+\tilde{\gamma}_{i, a} x_{a}^{2}\right)\right. \\
& \left.-\sum_{i=1, i \neq a}^{K} \ln \left(1+\sum_{j=1, j \notin\{i, a\}}^{K} \tilde{\gamma}_{i, j} x_{j}^{2}+\tilde{\gamma}_{i, a} x_{a}^{2}\right)\right),
\end{aligned}
$$

where $\tilde{\gamma}_{i, j}=\gamma_{i, j} \forall i \neq j, \tilde{\gamma}_{i, i}=\frac{e}{2 \pi} \gamma_{i, i} \forall i$.
It can be noticed that $\eta_{\mathrm{SE}}\left(x_{a}\right)$ is the sum of functions of the following form: $\ln \left(\alpha+\gamma x^{2}\right)$ and $-\ln \left(\beta+\gamma x^{2}\right)$ where both of them can be decomposed into difference of two concave/convex (DC) functions of the following forms $-\rho / 2 x^{2}-\left(-\rho / 2 x^{2}-\ln \left(\alpha+\gamma x^{2}\right)\right)$ and $-\bar{\rho} / 2 x^{2}-$ $\left(-\bar{\rho} / 2 x^{2}+\ln \left(\beta+\gamma x^{2}\right)\right)$ respectively, with $\rho \geq \frac{\gamma}{4 \alpha}$ and $\bar{\rho} \geq 2 \gamma$ [13]. Therefore, (P2) is a DC programming problem that can be solved by successive convex approximation (SCA) where the following optimization problem is solved in each SCA iteration:

$$
\begin{array}{ll}
\text { (P3) } \max _{x_{a}} & \tilde{\eta}_{\mathrm{SE}}\left(x_{a}\right) \\
\text { subject to } & x_{\min , a} \leq x_{a} \leq x_{\max , a}
\end{array}
$$

where $\max _{x_{a}}$ is written as

$$
\begin{aligned}
& \tilde{\eta}_{\mathrm{SE}}\left(x_{a}\right)=\sum_{i=1}^{K}\left(-\frac{\rho_{a, i} x_{a}^{2}}{2}-\left(-\frac{\rho_{a, i} x_{a, 0}^{2}}{2}-\ln \left(\Gamma_{a}+\tilde{\gamma}_{i, a} x_{a, 0}^{2}\right)\right.\right. \\
& \left.\left.-\left(\rho_{a, i} x_{a, 0}+\frac{2 \tilde{\gamma}_{i, a} x_{a, 0}}{\Gamma_{a}+\tilde{\gamma}_{i, a} x_{a, 0}^{2}}\right)\left(x_{a}-x_{a, 0}\right)\right)\right) /(2 \ln (2)) \\
& +\sum_{i=1, i \neq a}^{K}\left(-\frac{\bar{\rho}_{a, i} x_{a}^{2}}{2}-\left(-\frac{\bar{\rho}_{a, i} x_{a, 0}^{2}}{2}+\ln \left(\Gamma_{a, i}+\tilde{\gamma}_{i, a} x_{a, 0}^{2}\right)\right.\right. \\
& \left.\left.-\left(\bar{\rho}_{a, i} x_{a, 0}-\frac{2 \tilde{\gamma}_{i, a} x_{a, 0}}{\Gamma_{a, i}+\tilde{\gamma}_{i, a} x_{a, 0}^{2}}\right)\left(x_{a}-x_{a, 0}\right)\right)\right) /(2 \ln (2))
\end{aligned}
$$

with $\rho_{a, i}=\frac{\tilde{\gamma}_{i, a}}{4 \Gamma_{a}}, \bar{\rho}_{a, i}=2 \tilde{\gamma}_{i, a}, \Gamma_{a}=1+\sum_{j \neq a} \tilde{\gamma}_{i, j} x_{j}^{2}, \Gamma_{a, i}=$ $1+\sum_{j \neq a, j \neq i} \tilde{\gamma}_{i, j} x_{j}^{2}$ and $x_{a, 0}$ is the solution obtained in the previous SCA iteration.

The solution of the previous problem can be obtained in a closed form as

$x_{a}^{*}=\max \left(\min \left(x_{\mathrm{cr}}, x_{\max , a}\right), x_{\min , a}\right)$,

where $x_{\mathrm{cr}}$ is found to be

$x_{\mathrm{cr}}=\frac{x_{a, 0} \sum_{i=1}^{K}\left(\rho_{a, i}+\frac{2 \tilde{\gamma}_{i, a}}{\Gamma_{a}+\tilde{\gamma}_{i, a} x_{a, 0}^{2}}+\bar{\rho}_{a, i}-\frac{2 \tilde{\gamma}_{i, a}}{\Gamma_{a, i}+\tilde{\gamma}_{i, a} x_{a, 0}^{2}}\right)}{\sum_{i=1}^{K} \rho_{a, i}+\sum_{i \neq a} \bar{\rho}_{a, i}}$.

Based on the previous discussion, our proposed solution procedure for $(\mathbf{P} 1)$ proceeds as shown in Algorithm I. The optimization is done over two nested phases, where in the outer phase we solve a customized user-version of (P2) that is formulated based on the solution of the previous outer phase iterations with the aim of optimizing $(\mathbf{P} 1)$. In the inner phase of the solution procedure, the solution of each version of (P2) is obtained by successively solving different SCA-versions of (P3) using (11).

1) Feasibility problem: Before solving the SE maximization problem, we need to examine the problem feasibility. One 


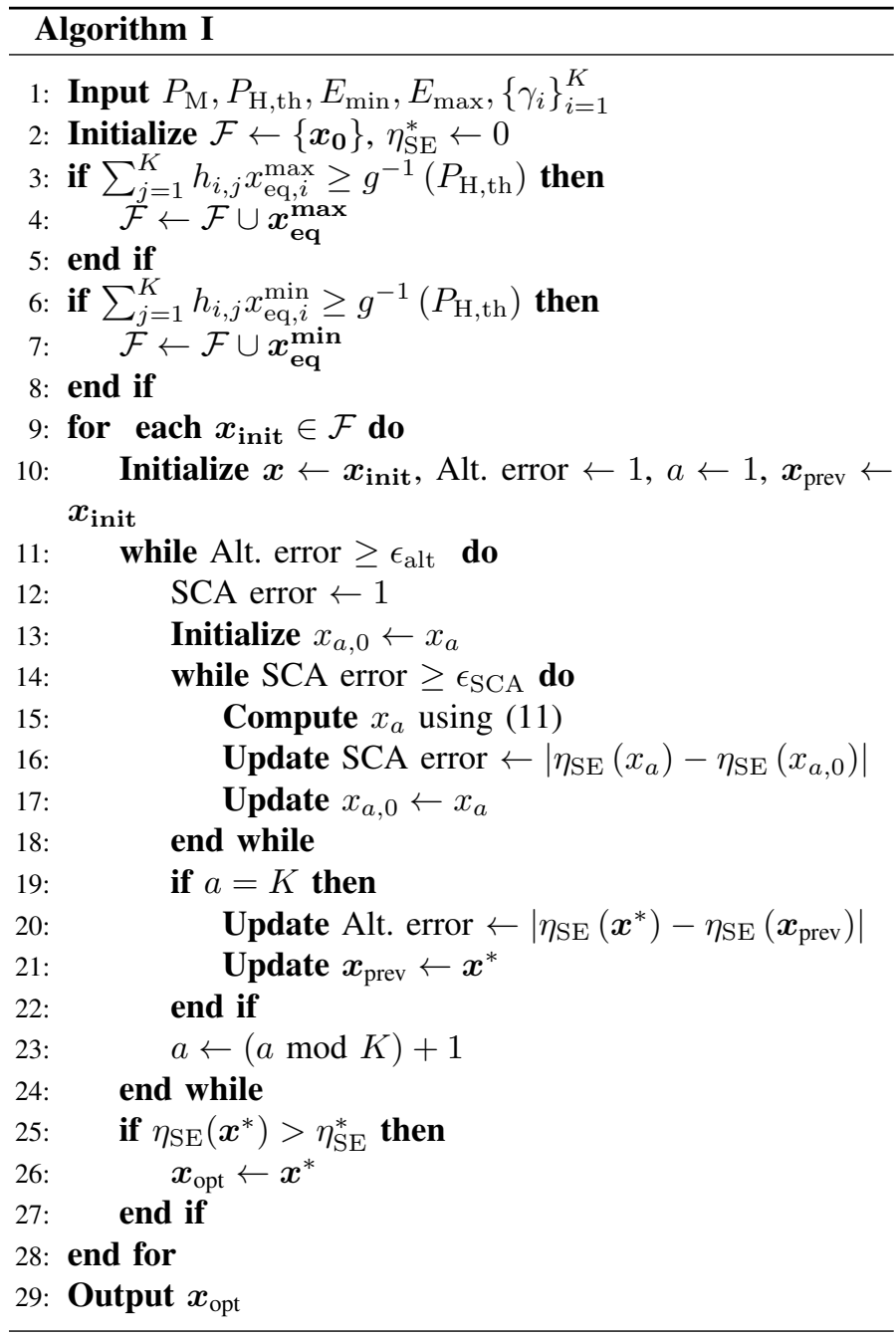

way to do so is to solve the following simplified optimization problem:

$$
\begin{array}{ll}
\min _{\boldsymbol{x}} & \kappa \sum_{i=1}^{K} x_{i}^{2} \\
\text { subject to } & \mathrm{C} 2, \mathrm{C} 3, \mathrm{C} 4 .
\end{array}
$$

After solving (P4), it can be deduced that $(\mathbf{P} 1)$ is infeasible if (P4) is infeasible or the optimal value of $(\mathbf{P} 4)$ exceeds $P_{\mathrm{M}}$. (P4) is a convex quadratic programming problem, thus we can solve it using convex optimization subroutines such as MATLAB CVX package [14].

2) Choosing initial point: Since the algorithm performance varies with the starting point, so we solve the problem using three possible initial points and take the best solution. The possible points are:

1) $\boldsymbol{x}_{\mathrm{eq}}^{\min }: x_{\mathrm{eq}, i}^{\min }=\frac{E_{\min } R_{\mathrm{PD}}}{\eta_{\phi} h_{\mathrm{min}}} \forall i$, which is the equal allocation associated with the minimum illumination constraint. This proposed starting point gives good solution to the dense cell deployment scenario.
TABLE I: Simulation Parameters.

\begin{tabular}{|c|c|c|}
\hline$N_{0}=10^{-21} \mathrm{~W} / \mathrm{Hz}$ & $B_{\mathrm{v}}=20 \mathrm{MHz}$ & $H=3 \mathrm{~m}$ \\
\hline$P_{M}=450 \mathrm{~W}$ & $K=9$ & $E_{\min }=100 \operatorname{lux}$ \\
\hline$A_{\mathrm{PD}}=1 \mathrm{~cm}^{2}$ & $R_{\mathrm{PD}}=0.6 \mathrm{~A} / \mathrm{W}$ & $d_{\min }=0.5 \mathrm{~m}$ \\
\hline$\eta_{\phi}=7500$ lumens $/ \mathrm{A}$ & $\kappa=23 \mathrm{~W} / \mathrm{A}^{2}$ & $\phi_{\mathrm{A}}=60^{\circ}$ \\
\hline$E_{\max }=3000$ lux & $h_{\mathrm{T}}=1 \mathrm{~m}$ & \\
\hline
\end{tabular}

2) $\boldsymbol{x}_{\mathrm{eq}}^{\min }: x_{\mathrm{eq}, i}^{\max }=\min \left(\sqrt{\frac{P_{\mathrm{M}}}{\kappa K}}, \frac{E_{\max } R_{\mathrm{PD}}}{\eta_{\phi} h_{\max }\left|\mathcal{N}_{i}\right|}\right) \forall i$, which is the equal allocation associated with the maximum illumination constraint ${ }^{2}$. This initial point gives a good solution to the sparse cell deployment scenario.

3) $x_{0}$ : the solution of (P4), which becomes important when two previous solutions do not satisfy $\mathrm{C} 4$.

\section{B. Energy harvesting maximization}

In this study, we are interested in studying the energy harvesting performance limits of SLIPT systems in interference scenario under illumination constraints. To this end, we formulate the following problem

$$
\begin{array}{ll}
\max _{\boldsymbol{x}} & P_{\mathrm{H}, \text { tot }} \\
\text { subject to } & \mathrm{C} 1, \mathrm{C} 2, \mathrm{C} 3 .
\end{array}
$$

This problem is not a convex program, consequently, we propose using the following lower bound

$$
\begin{aligned}
\tilde{P}_{\mathrm{H}, \mathrm{tot}}=\sum_{i=1}^{K} \ln \left(\sum_{j=1}^{K} 0.75 h_{i, j} x_{j} \mathrm{~V}_{\mathrm{t}}\right) \\
+\sum_{i=1}^{K} \ln \left(\ln \left(1+\sum_{i=1}^{K} h_{i, j} x_{j} / I_{o}\right)\right)
\end{aligned}
$$

as a surrogate function for the original one and solve the following optimization problem instead

(P6)

$$
\begin{array}{ll}
\max _{\boldsymbol{x}} & \tilde{P}_{\mathrm{H}, \text { tot }} \\
\text { subject to } & \mathrm{C} 1, \mathrm{C} 2, \mathrm{C} 3 .
\end{array}
$$

The objective function $\tilde{P}_{\mathrm{H}, \mathrm{tot}}(\boldsymbol{x})$ is a sum of either the composition of $\ln ($.$) or \ln (\ln ()$.$) function, which are both concave$ functions, with affine combination of the optimization variables. Thus, $\tilde{P}_{\mathrm{h} \text {,tot }}(\boldsymbol{x})$ is a concave function, which results in a convex optimization problem thanks to the convex set properties of C1 - C3 [15]. As a result, (P6) can be solved using any of the available convex programming software packages.

\section{Simulation Results}

The simulation setup consists of nine transmitters distributed over a square lattice on the ceiling of an indoor area at a height $H$ from the ground. It is assumed that user-cell association is done based on nearest distance, which is obviously translated to the square cell boundaries of side length $d_{\min }$ in the receivers

\footnotetext{
${ }^{2}\left|\mathcal{N}_{i}\right|$ represents the cardinality of the set $\mathcal{N}_{i}$ (number of transmitters in cluster $i$ which is constant due to uniform lattice layout of transmitters and equals 4 for square grid lattice)
} 
plane as shown inf Fig. 1. The nine users served by the system are distributed at random such that each user is placed according to an uniform distribution within its corresponding transmitter square cell boundary in the receivers plane. Moreover, the receivers-to-ground vertical separation is assumed to be $h_{\mathrm{T}}$, with all transmitters and receivers having horizontal orientation. The average results presented in this work are calculated based on 1000 random users' locations realizations. The simulation parameters values are provided in Table I, unless otherwise specified. Throughout the following simulations, we study the performance of three allocation strategies, namely maximized SE solution (solution of (P1)), maximized harvested energy solution (solution of (P6)), and the basic uniform allocation $\left(\boldsymbol{x}_{\mathrm{eq}}^{\max }\right)$. We assume zero SE and energy harvesting performance for infeasible problem realizations in average calculation of these objectives.

In the first simulation, we study the effect of varying the distance between neighboring cells (which is the main controller for interference severity) on average optimized SE and average optimized harvested energy. To monitor the extreme SE and total harvested energy performances, we keep the per-user harvested power constraint inactive in this simulation. As $d_{\min }$ increases, it can be seen in Fig. 2 that average SE performance is improved because interference terms of the objective function gets smaller and this improvement outweighs the performance deterioration that could be caused as the direct links channel gains gets worsened for small $d_{\min }$ (probability of increased separation between users and their associated transmitters as cells gets larger). Thus, it can be deduced that if $d_{\min }$ increases to values higher than $3.5 \mathrm{~m}$, the average optimized SE performance will deteriorate and even reach zero as more realizations will become infeasible due to minimum illumination constraint. As for the relative performance between the three solutions, it can be noticed that the SE performance loss due to maximizing harvested energy diminishes as $d_{\min }$ increases. However, the SE performance gap between maximized SE solution and the two other solutions is significantly high in dense deployment scenarios. On the other hand, the average total harvested power performance of the three considered solutions gets worse as $d_{\text {min }}$ gets larger as shown in Fig. 3, which is expected since the receivers get further away from the transmitters so that the effective areas of their receivers are exposed to smaller power densities. It can be seen that the maximized harvested energy solution outperforms the two other solutions, and the performance gap between the three algorithms gets smaller as $d_{\min }$ increases. Interestingly, it was found that, from harvested energy perspective, the uniform allocation solution outperforms SE maximization for small values of $d_{\min }$ and the effect is reversed for large values. It can be deduced that energy harvesting solution tends to always satisfy $\mathrm{C} 1$ with equality as the $P_{\mathrm{H}, \text { tot }}$ is monotonically increasing with the power allocation. However, this is not the case for SE maximization which could easily decrease the total transmitted power to alleviate interference effect. Thus, at small $d_{\min }$, the uniform allocation solution pushes transmitters to fully use $P_{\mathrm{M}}$ which makes it better

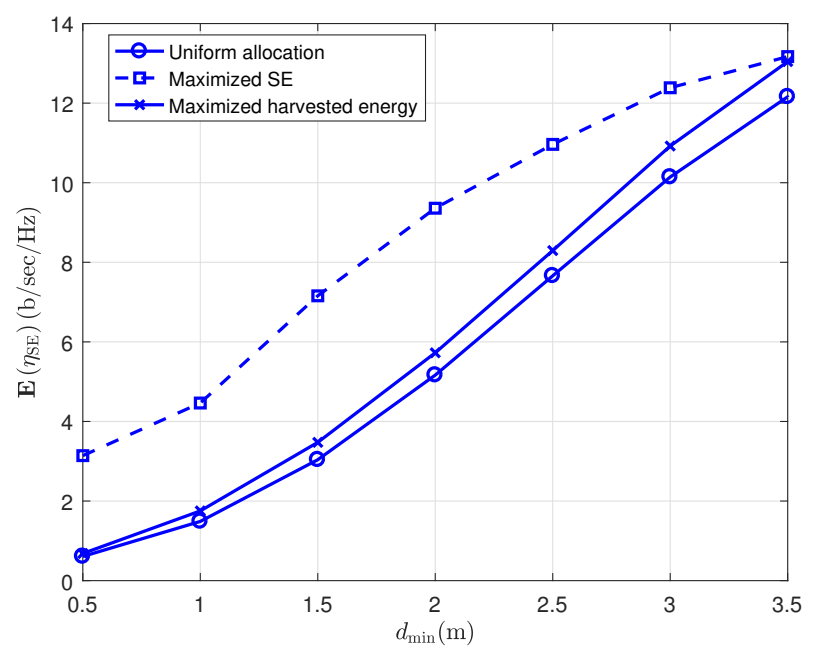

Fig. 2: Average SE vs Min. inter-cell separation distance $d_{\min }$

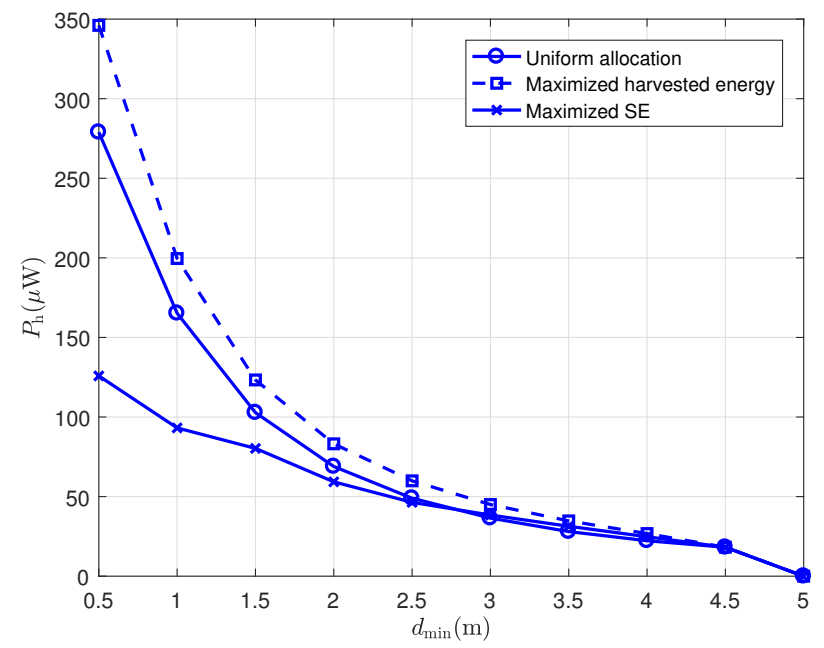

Fig. 3: Average harvested power vs Min. inter-cell separation distance $d_{\min }$

than the maximized SE solution. Albeit, when $d_{\min }$ becomes large enough, the maximized SE solution exhausts $P_{\mathrm{M}}$ while allocating power in a way that considers channel asymmetries which is not considered by the uniform allocation. It can be seen clearly from Fig. 2 and 3 that maximizing one of the objectives comes at the cost of significant losses for the other, specially under severe interference conditions.

In the second simulation, we study the effect of increasing the minimum harvested power constraint on the average SE performance for $E_{\max }=5000$ lux. It can be noticed that as $P_{\mathrm{H}, \text { th }}$ increases the SE performance of optimized SE solution is not affected initially (C4 is not active yet). As $P_{\mathrm{H} \text {,th }}$ continues increasing, the SE performance deteriorates as the feasibility space gets tighter and the optimization solution satisfies $\mathrm{C} 4$ with equality. As $P_{\mathrm{H}, \text { th }}$ keeps increasing, the probability of 


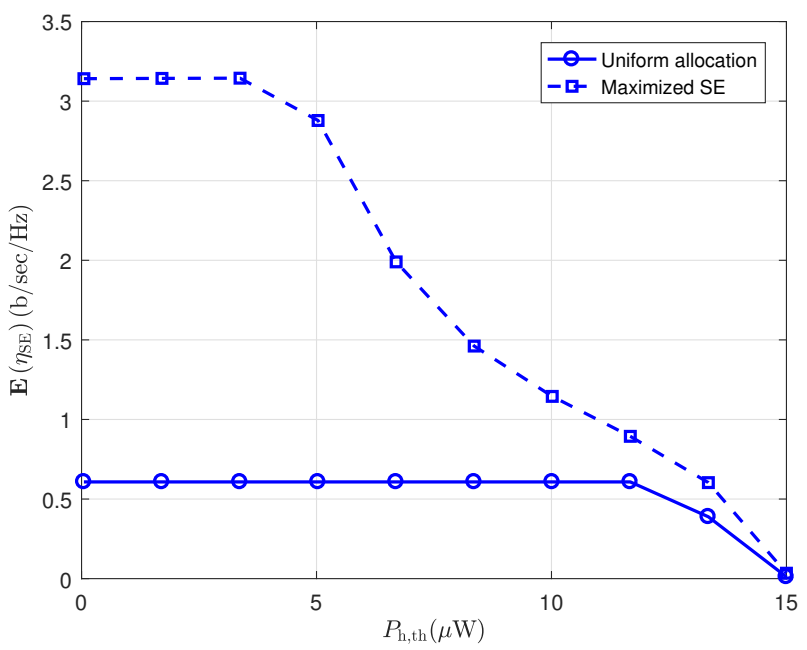

Fig. 4: Average SE vs $P_{\mathrm{H}, \mathrm{th}}$

having more infeasible problems with zero contribution to the average SE performance increases. On the other hand, uniform allocation solution maintains SE constant as $P_{\mathrm{H}, \mathrm{th}}$ increases till it reaches a critical value where the uniform allocation solution infeasibility frequency increases, hence zero SE starts to be counted for a subset of the realizations which deteriorates the average till it reaches zero.

\section{CONCLUSION}

In this paper, we considered the power allocation problem for a multi-cell SLIPT system where interference has a nonnegligible effect on the system performance. We investigated the performance limits of this system by maximizing two of its design objectives, namely system overall SE, and total harvested energy severally under lighting constraints. Moreover, we monitored the effect of optimizing each objective in terms of optimality losses in the other through extensive simulations. Furthermore, we studied the effect of adding minimum energy harvesting constraint per user on the SE performance metric and proposed a distributed algorithm to solve this problem. Simulation results highlighted the trade-off between SE and energy harvesting whose severity explodes as cells becomes in close proximity. These results motivated studying the SE- energy harvesting multi-objective optimization problem in order to reach solutions that achieve balance between the two design objectives. This approach would give SLIPT system designers the freedom to find proper allocation that accounts for the desired importance of each design objective.

\section{REFERENCES}

[1] M. A. Khalighi and M. Uysal, "Survey on free space optical communication: A communication theory perspective," IEEE Commun. Surveys Tuts., vol. 16, no. 4, pp. 2231-2258, Jun. 2014.

[2] C.-X. Wang, F. Haider, X. Gao, X.-H. You, Y. Yang, D. Yuan, H. Aggoune, H. Haas, S. Fletcher, and E. Hepsaydir, "Cellular architecture and key technologies for 5G wireless communication networks," IEEE Commun. Mag., vol. 52, no. 2, pp. 122-130, Feb. 2014.

[3] M. R. Zenaidi, Z. Rezki, M. Abdallah, K. A. Qaraqe, and M. S. Alouini, "Achievable rate-region of VLC/RF communications with an energy harvesting relay," in IEEE Global Commun. Conf. (GLOBECOM), Dec. 2017, pp. 1-7.

[4] T. Rakia, H. C. Yang, F. Gebali, and M. S. Alouini, "Optimal design of dual-hop VLC/RF communication system with energy harvesting," IEEE Commun. Lett., vol. 20, no. 10, pp. 1979-1982, Oct. 2016.

[5] A. M. Abdelhady, O. Amin, A. Chaaban, and M. S. Alouini, "Resource allocation for outdoor visible light communications with energy harvesting capabilities," in IEEE Globecom Workshops (GC Wkshps), Singapore, Dec. 2017, pp. 1-6.

[6] Y. Li, N. Huang, J. Wang, Z. Yang, and W. Xu, "Sum rate maximization for VLC systems with simultaneous wireless information and power transfer," IEEE Photon. Technol. Lett., vol. 29, no. 6, Mar. 2017.

[7] A. M. Abdelhady, O. Amin, A. Chaaban, and M. S. Alouini, "Spectralefficiency - illumination pareto front for energy harvesting enabled VLC system." [Online]. Available: http://hdl.handle.net/10754/626362

[8] P. D. Diamantoulakis, G. K. Karagiannidis, and Z. Ding, "Simultaneous lightwave information and power transfer (SLIPT)," IEEE Trans.Green Commun. Netw., Doi: 10.1109/TGCN.2018.2818325 2018.

[9] Y. Liu, H. Y. Chen, K. Liang, C. W. Hsu, C. W. Chow, and C. H. Yeh, "Visible light communication using receivers of camera image sensor and solar cell," IEEE Photon. J., vol. 8, no. 1, pp. 1-7, Feb. 2016.

[10] Z. Wang, D. Tsonev, S. Videv, and H. Haas, "On the design of a solarpanel receiver for optical wireless communications with simultaneous energy harvesting," vol. 33, no. 8, pp. 1612-1623, Aug. 2015.

[11] J. M. Kahn and J. R. Barry, "Wireless infrared communications," Proc. IEEE, vol. 85, no. 2, pp. 265-298, Feb. 1997.

[12] A. Lapidoth, S. M. Moser, and M. Wigger, "On the capacity of free-space optical intensity channels," IEEE Trans. Inf. Theory, vol. 55, no. 10, pp. 4449-4461, Oct. 2009.

[13] A. M. Abdelhady, O. Amin, A. Chaaban, and M. S. Alouini, "Downlink resource allocation for multichannel TDMA visible light communications," in 2016 IEEE Global Conf. Signal Inform. Process. (GlobalSIP), Greater Washington, D.C., USA, Dec. 2016, pp. 1-5.

[14] M. Grant and S. Boyd, "CVX: Matlab software for disciplined convex programming, version 2.1," http://cvxr.com/cvx, Mar. 2014.

[15] S. Boyd and L. Vandenberghe, Convex optimization. Cambridge university press, 2004. 